\title{
Resolution enhancement in computerized tomographic imaging
}

\author{
Hsin M. Shieh, ${ }^{1, *}$ Chih-Hung Chung, ${ }^{1}$ and Charles L. Byrne ${ }^{2}$ \\ 'Department of Electrical Engineering, Feng Chia University, 100 Wenhwa Road, Seatwen, Taichung, Taiwan 40724 \\ ${ }^{2}$ Department of Mathematical Sciences, University of Massachusetts Lowell, \\ One University Avenue, Lowell, Massachusetts 01854, USA \\ ${ }^{*}$ Corresponding author: hmshieh@fcu.edu.tw
}

Received 28 January 2008; revised 29 May 2008; accepted 7 July 2008; posted 9 July 2008 (Doc. ID 91312); published 28 July 2008

\begin{abstract}
We consider the problem of reconstructing an object function $f(r)$ from finitely many linear functional values. In our main application, the function $f(r)$ is a tomographic image, and the data are integrals of $f(r)$ along thin strips. Because the data are limited, resolution can be enhanced through the inclusion of prior knowledge. One way to do that, a generalization of the prior discrete Fourier transform (PDFT) method, was suggested in 1982 [SIAM J. Appl. Math. 42, 933 (1982)] but was found to be difficult to implement for the tomography problem, and that application was not pursued. Recent advances in approximating the PDFT make it possible to achieve the desired resolution enhancement in an easily implemented procedure. (C) 2008 Optical Society of America

OCIS codes: $\quad 100.3010,100.3020,100.3190,100.6640$
\end{abstract}

\section{Introduction}

The problem is to reconstruct a (possibly complexvalued) function $f: R^{D} \rightarrow C$ from finitely many linear functional measurements $d_{n}, n=1, \ldots, N$, pertaining to $f$. The function $f(r)$ represents the physical object of interest, such as the spatial distribution of acoustic energy in sonar, the distribution of x-ray-attenuating material in transmission tomography, the distribution of radionuclides in emission tomography, and the sources of reflected radio waves in radar. Often the reconstruction, or estimate, of the function $f$ takes the form of an image in two or three dimensions; for that reason, we also speak of the problem as one of image reconstruction. The data are obtained through measurements. Because there are only finitely many measurements, the problem is highly underdetermined, and even noise-free data are insufficient to specify a unique solution.

The function $f(r)$ of interest to us here has finite support, that is, there is a closed, bounded set

0003-6935/08/224116-05\$15.00/0

(C) 2008 Optical Society of America
$S \subseteq R^{D}$ such that $f(r)=0$ for $r$ not in $S$; we may or may not know $S$ precisely. The measurements of interest to us are linear; that is, there are known functions $h^{n}(r), n=1, \ldots, N$, with

$$
d_{n}=\int_{S} f(r) \overline{h^{n}(r)} \mathrm{d} r
$$

We shall assume that the set $\left\{h^{n}(r), n=1, \ldots, N\right\}$ is linearly independent. A standard method for estimating $f(r)$ involves formulating the problem in a Hilbert-space setting.

The reconstruction approach described in this paper was designed initially to reconstruct a function from finitely many values of its Fourier transform. Because it generalized the discrete Fourier transform and incorporated prior knowledge, it was called the prior discrete Fourier transform (PDFT) [1,2]. The possibility was raised in [1] of applying the PDFT approach to the reconstruction of images from tomographic data, but the PDFT, as then computed, was impractical for such large problems, so that avenue was left unexplored. Recent improvements $[3,4]$ that make it possible to calculate efficiently a discretized 
PDFT estimate suggest that now is a good time to reopen that line of enquiry.

\section{Hilbert Space Reconstruction Methods}

In Sections 2-4 we present a rigorous discussion of the reconstruction problem in Hilbert space.

In many applications the data are related linearly to $f$. To model the operator that transforms $f$ into the data vector, we need to select an ambient space containing $f$. Typically, we choose a Hilbert space. The selection of the inner product provides an opportunity to incorporate prior knowledge about $f$ into the reconstruction. The inner product induces a norm, and our reconstruction is that function, consistent with the data, for which this norm is minimized.

Minimum-Norm Solutions. Our estimation problem is highly underdetermined; there are infinitely many functions in $L^{2}(S)$ that are consistent with the data and might be the right answer. Such underdetermined problems are often solved by acting conservatively and selecting as the estimate of that function consistent with the data that has the smallest norm. At the same time, however, we often have some prior information about $f$ that we would like to incorporate into the estimate. One way to achieve both of these goals is to select the norm to incorporate prior information about $f$ and then to take as the estimate of $f$ the function, consistent with the data, for which the chosen norm is minimized.

The data vector $\mathbf{d}=\left(d_{1}, \ldots, d_{N}\right)^{T}$ is in $C^{N}$, and the linear operator $\mathcal{H}$ from $L^{2}(S)$ to $C^{N}$ takes $f$ to d; so we write $\mathbf{d}=\mathcal{H} f$. Associated with the mapping $\mathcal{H}$ is its adjoint operator, $\mathcal{H}^{\dagger}$, going from $C^{N}$ to $L^{2}(S)$ and given, for each vector $\mathbf{a}=\left(a_{1}, \ldots, a_{N}\right)^{T}$, by

$$
\left(\mathcal{H}^{\dagger} \mathbf{a}\right)(r)=a_{1} h_{1}(r)+\ldots+a_{N} h_{N}(r) .
$$

The operator from $C^{N}$ to $C^{N}$ defined by $\mathcal{H H}^{\dagger}$ corresponds to an $N \times N$ matrix, which we shall also denote $\mathcal{H} \mathcal{H}^{\dagger}$. If the functions $h^{n}(r)$ are linearly independent, then this matrix is positive definite, therefore invertible.

Given the data vector $\mathbf{d}$, we can solve the system of linear equations

$$
\mathbf{d}=\mathcal{H} \mathcal{H}^{\dagger} \mathbf{a}
$$

for the vector a. Then the function

$$
f^{0}(r)=\left(\mathcal{H}^{\dagger} \mathbf{a}\right)(r)
$$

is consistent with the measured data and is the function in $L^{2}(S)$ of least norm for which this is true. The function $u(r)=f(r)-f^{0}(r)$ has the property $\mathcal{H} u=0$. It is easy to see that

$$
\|f\|_{2}^{2}=\left\|f^{0}\right\|_{2}^{2}+\|u\|_{2}^{2} .
$$

The estimate $f^{0}(r)$ is the minimum-norm solution, with respect to the usual $L^{2}(S)$ norm. If we change the norm on $L^{2}(S)$, or, equivalently, the inner product, then the minimum-norm solution will change.

For any continuous linear operator $\mathcal{T}$ on $L^{2}(S)$, the adjoint operator, denoted $\mathcal{T}^{\dagger}$, is defined by

$$
\langle\mathcal{T} f, h\rangle_{2}=\left\langle f, \mathcal{T}^{\dagger} h\right\rangle_{2} .
$$

The adjoint operator will change when we change the inner product.

\section{Class of Inner Products}

Let $\mathcal{T}$ be a continuous, linear and invertible operator on $L^{2}(S)$. Define the $\mathcal{T}$ inner product to be

$$
\langle f, h\rangle_{\mathcal{T}}=\left\langle\mathcal{T}^{-1} f, \mathcal{T}^{-1} h\right\rangle_{2} .
$$

We can then use this inner product to define the problem to be solved. We now say that

$$
d_{n}=\left\langle f, t^{n}\right\rangle_{\mathcal{T}},
$$

for known functions $t^{n}(r)$. Using the definition of the $\mathcal{T}$ inner product, we find that

$$
d_{n}=\left\langle f, h^{n}\right\rangle_{2}=\left\langle\mathcal{T} f, \mathcal{T} h^{n}\right\rangle_{\mathcal{T}}
$$

The adjoint operator for $\mathcal{T}$, with respect to the $\mathcal{T}$ norm, is denoted $\mathcal{T}^{*}$, and is defined by

$$
\langle\mathcal{T} f, h\rangle_{\mathcal{T}}=\left\langle f, \mathcal{T}^{*} h\right\rangle_{\mathcal{T}} .
$$

Therefore,

$$
d_{n}=\left\langle f, \mathcal{T}^{*} \mathcal{T} h^{n}\right\rangle_{\mathcal{T}} .
$$

Lemma 3.1. We have $\mathcal{T}^{*} \mathcal{T}=\mathcal{T}^{\dagger}$.

Consequently, we have

$$
d_{n}=\left\langle f, \mathcal{T} \mathcal{T}^{\dagger} h^{n}\right\rangle_{\mathcal{T}} .
$$

\section{Minimum- $\mathcal{T}$-Norm Solutions}

The function $f^{1}$ consistent with the data and having the smallest $\mathcal{T}$ norm has the algebraic form

$$
f^{1}=\sum_{m=1}^{N} c_{m} \mathcal{T} \mathcal{T}^{\dagger} h^{m} .
$$

Applying the $\mathcal{T}$ inner product to both sides of Eq. (13), we get

$$
\begin{gathered}
d_{n}=\left\langle f^{1}, \mathcal{T} \mathcal{T}^{\dagger} h^{n}\right\rangle_{\mathcal{T}} \\
=\sum_{m=1}^{N} c_{m}\left\langle\mathcal{T} \mathcal{T}^{\dagger} h^{m}, \mathcal{T} \mathcal{T}^{\dagger} h^{n}\right\rangle_{\mathcal{T}} .
\end{gathered}
$$

Therefore, 


$$
d_{n}=\sum_{m=1}^{N} c_{m}\left\langle\mathcal{T}^{\dagger} h^{m}, \mathcal{T}^{\dagger} h^{n}\right\rangle_{2} .
$$

We solve this system for the $c_{m}$ and insert them into Eq. (13) to get our reconstruction. The Gram matrix that appears in Eq. (16) is positive definite, but is often ill conditioned; increasing the main diagonal by a percent or so usually is sufficient regularization.

We turn now to an important case in which the measured data are values of the Fourier transform of the function $f$.

\section{Case of Fourier Transform Data}

To illustrate these minimum- $\mathcal{T}$-norm solutions, we consider the case in which the data are values of the Fourier transform of $f$. Specifically, suppose that

$$
d_{n}=\int_{S} f(x) e^{-i \omega_{n} x} \mathrm{~d} x,
$$

for arbitrary values $\omega_{n}$.

\section{A. $L^{2}(-\pi, \pi)$ Case}

Assume that $f(x)=0$, for $|x|>\pi$. Then $S=[-\pi, \pi]$. The minimum-2-norm solution in $L^{2}(S)$ has the form

$$
f^{0}(x)=\sum_{m=1}^{N} a_{m} e^{i \omega_{m} x}
$$

with

$$
d_{n}=\sum_{m=1}^{N} a_{m} \int_{-\pi}^{\pi} e^{i\left(\omega_{m}-\omega_{n}\right) x} \mathrm{~d} x
$$

For the equispaced values $\omega_{n}=n$ we find that $a_{m}=$ $d_{m}$ and the minimum-norm solution is the discrete Fourier transform

$$
f^{0}(x)=\sum_{n=1}^{N} d_{n} e^{i n x}
$$

\section{B. Oversampled Case}

Suppose that $f(x)=0$ for $|x|>X$, where $0<X<\pi$. Then we use $L^{2}(-X, X)$ as the Hilbert space, and $S=[-X, X]$. For equispaced data at $\omega_{n}=n$, we have

$$
d_{n}=\int_{-\pi}^{\pi} f(x) \chi_{X}(x) e^{-i n x} \mathrm{~d} x
$$

so that the minimum-norm solution has the form

$$
f^{0}(x)=\chi_{X}(x) \sum_{m=1}^{N} a_{m} e^{i m x}
$$

with

$$
d_{n}=2 \sum_{m=1}^{N} a_{m} \frac{\sin X(m-n)}{m-n} .
$$

The minimum-norm solution is supported on $[-X, X]$ and consistent with the Fourier-transform data.

\section{Using a Prior Estimate of $f$}

Suppose that $f(x)=0$ for $|x|>\pi$ again, and that $p(x)$ satisfies

$$
0<\epsilon \leq p(x) \leq E<+\infty,
$$

for all $x$ in $S=[-\pi, \pi]$. Define the operator $\mathcal{T}$ by $(\mathcal{T} f)(x)=\sqrt{p(x)} f(x)$. The $\mathcal{T}$ norm is then

$$
\langle f, h\rangle_{\mathcal{T}}=\int_{-\pi}^{\pi} f(x) \overline{h(x)} p(x)^{-1} \mathrm{~d} x .
$$

It follows that

$$
d_{n}=\int_{-\pi}^{\pi} f(x) p(x) e^{-i n x} p(x)^{-1} \mathrm{~d} x
$$

so that the minimum $\mathcal{T}$ norm solution is

$$
f^{1}(x)=\sum_{m=1}^{N} c_{m} p(x) e^{i m x}=p(x) \sum_{m=1}^{N} c_{m} e^{i m x},
$$

where

$$
d_{n}=\sum_{m=1}^{N} c_{m} \int_{-\pi}^{\pi} p(x) e^{i(m-n) x} \mathrm{~d} x .
$$

The estimate $f^{1}(x)$ is $f^{w}(x)$, in the terminology of a previous section. If we have prior knowledge about the support of $f$, or some idea of its shape, we can incorporate that prior knowledge into the reconstruction through the choice of $p(x)$.

The reconstruction in Eq. (27) was presented in [1], where it was called the PDFT method. The PDFT was based on an earlier noniterative version of the Gerchberg-Papoulis band-limited extrapolation procedure [5]. The PDFT was then applied to image reconstruction problems in [2]. An application of the PDFT was presented in [6]. In [7] we extended the PDFT to a nonlinear version, the indirect PDFT, that generalizes Burg's maximum entropy spectrum estimation method. The PDFT was applied to the phase problem in [8] and in [9] both the PDFT and indirect PDFT were examined in the context of Wiener filter approximation. More recent work on these topics is discussed in the book [10].

\section{Case of Tomographic Data}

The ideal model for transmission tomography is that the data we have are line integrals through the attenuation function $f(r)$, from which we must estimate $f(r)$. In practice, of course, what we have measured will not be precisely line integrals, but more like strip 
integrals, and, in any case, we will have finitely many of them. We shall consider here only the case $D=2$; that is, the function $f(r)$ is a function of two real variables. We take each $h^{n}(r)$ to be the characteristic function of a strip, that is, $h^{n}(r)=1$ for points $r$ within the strip, and $h^{n}(r)=0$ for $r$ outside the strip. By a strip we mean the set of all points that lie within $\delta>0$ of a line segment through the domain of $f(r)$, where $\delta$ is fixed and small. In the examples to be discussed later, the function $f(r)$ is a simulated slice of a human head. We take the prior function $p(r)$ to have the value 1 within the oval describing the support of the head slice, and $\epsilon>0$ outside the support. The use of a small $\epsilon>0$ instead of zero serves to introduce a small amount of regularization and to make the reconstruction less sensitive to noise. The function $f(r)$ is discretized into finitely many pixel values, the PDFT is approximated by the DPDFT, and the algebraic reconstruction technique (ART) is used to find the minimum-weighted-norm solution.

\section{The Discrete PDFT}

The basic idea in the discrete PDFT (DPDFT) approach is to discretize the functions $f(r), h^{n}(r)$, and $p(r)$, replacing the function $f(r)$ with the $J$ by one vector f with entries $f_{j}, h^{n}(r)$ with the $J$ by one vector $\mathbf{h}^{n}$ with entries $h_{j}^{n}$, and $p(r)$ with the $J$ by one vector $\mathbf{p}$ with entries $p_{j}>0$. Then the data values $d_{n}$ are related to the vector $\mathbf{f}$ by

$$
d_{n}=\sum_{j=1}^{J} f_{j} \overline{h_{j}^{n}} .
$$

In matrix notation, we write this system as $A \mathbf{f}=\mathbf{d}$, where $A_{n j}=\overline{h_{j}^{n}}$, and the entries of $\mathbf{d}$ are the $d_{n}$. We take $J>N$, so that this system of equations is underdetermined. By analogy with the PDFT, we now seek the vector f, satisfying the system in Eq. (29), for which the norm

$$
\|\mathbf{f}\|_{\mathbf{w}}=\sqrt{\sum_{j=1}^{J}\left|f_{j}\right|^{2} p_{j}^{-1}}
$$

is minimized.

Let $g_{j}=f_{j} / \sqrt{p_{j}}$ and $B_{n j}=A_{n j} \sqrt{p_{j}}$. Then $B \mathbf{g}=$ $A \mathbf{f}=\mathbf{d}$, and we seek the minimum Euclidean norm solution of the system $B \mathbf{g}=\mathbf{d}$. Iterative algorithms such as the ART produce this minimum-norm solution when the system is underdetermined and the initial vector is zero. Notice that we no longer need to calculate the Gram matrix, and the solution to the system is approximated iteratively, which is computationally feasible, even for large $N$.

\section{Simulations}

To test our algorithm we applied it to simulated transmission-tomographic data. For the purpose of accommodating more realistic computerized tomographic imaging applications, a simulated head phantom in Fig. 1(a) is considered as the object func- tion to be reconstructed in the simulation. The superior of the PDFT over the filtered backprojection (FBP) and the ART methods is especially evident.

In the data collection we sampled 151 projectiondata values at a uniform interval for each angle, and there were 90 different angles taken from $0^{\circ}$ to 0 to $180^{\circ}$, with a uniform step angle of $2^{\circ}$. The FBP estimate and the ART estimate after one iteration are shown in Figs. $\underline{1(b)}$ and 1 (c), respectively. In this example the PDFT cannot be calculated directly by using a personal computer because of the process of solving a large system of linear equations, like most realistic applications. Therefore, we turn to the iterative DPDFT algorithm. With the prior in Fig. 1(c), the DPDFT estimate after one iteration in Fig. 1(d) gives a better resolution than the FBP and ART estimates. To avoid what might otherwise be slow convergence of the iteration, a random access order of the equations is used to obtain the image results in Fig. 1(c) and 1(d) [4]. It is important to note that only a single pass through the equations was used to obtain the DPDFT images, which makes this approach even more computationally attractive.

For a quantitative evaluation, the root mean square error between the object function and its estimate can be essentially considered a measure of the accuracy of the image reconstruction. Since the DPDFT reconstructs the image only within the prior function's domain, the root mean square error calculated from the whole picture is not appropriate to be used for the comparison, and we take the root mean square error over the object function's true domain (RMSEOFTD) instead:

$$
\text { RMSEOFTD }=\sqrt{\frac{1}{N} \sum_{\mathfrak{D}}\left|o_{n}-r_{n}\right|^{2}},
$$

where $N$ is the total number of pixels over the object function's true domain $\mathfrak{D}$, and $o_{n}$ and $r_{n}$ each represents the pixel value of the object and the image, respectively. For the example in Fig. 1, RMSEOFTD for the FBP, ART, and DPDFT estimates is 63.036, 24.457 , and 19.547, respectively.

\section{Conclusions}

It has been known for some time that reconstruction from limited data can be improved through the use of prior information about the object being reconstructed. The PDFT is one effective way in which to incorporate prior information. Difficulties with implementing the PDFT on realistically large tomographic problems have been overcome through the use of the DPDFT approximation method. The DPDFT involves the calculation of a weighted-minimum-norm solution of a large system of linear equations, for which we have chosen to use the ART method. The ART itself was originally proposed for tomographic reconstruction [11]. The novelty here is in the use of the inverse of the prior estimate of the object as a weight in redefining the norm to be minimized. 

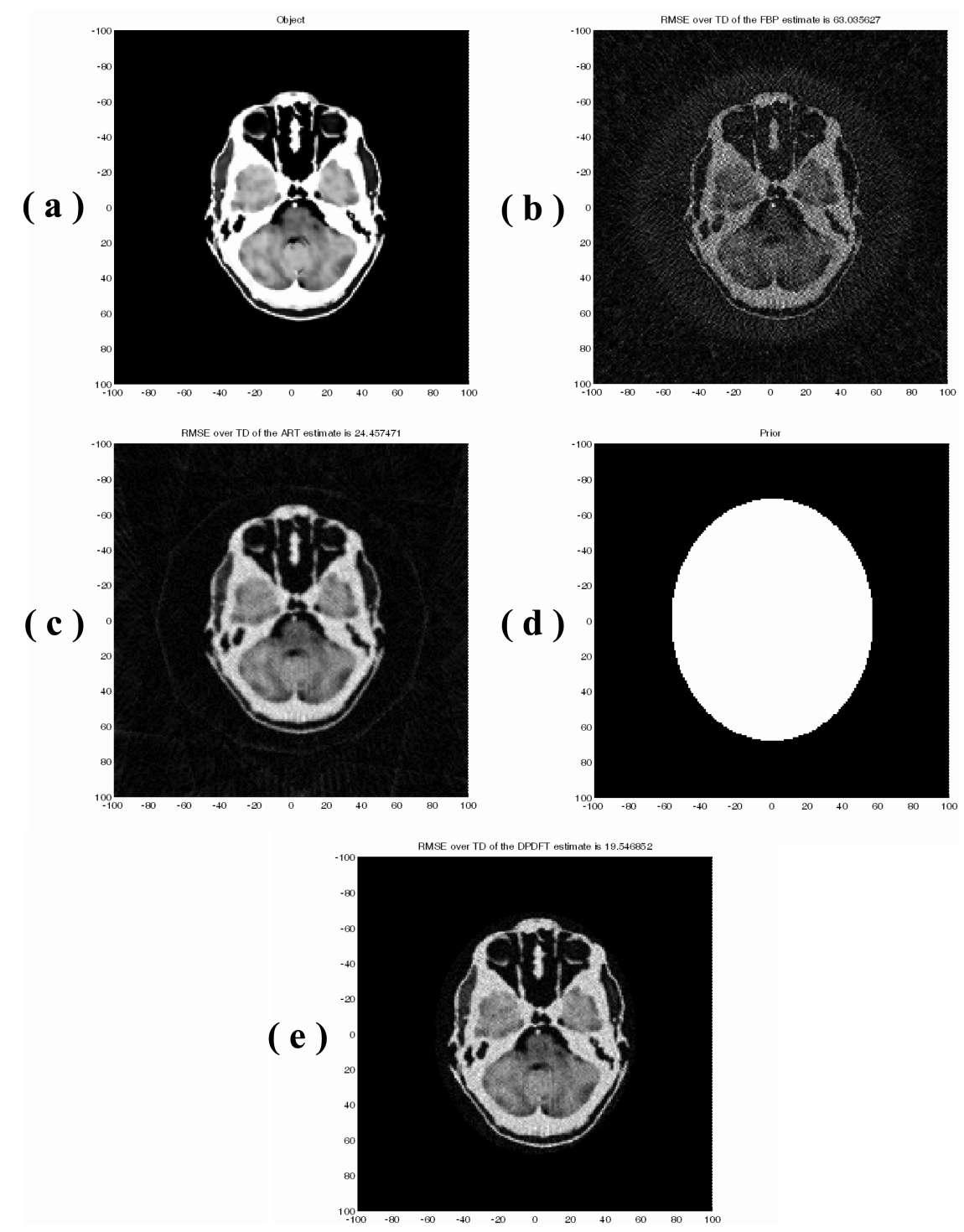

Fig. 1. Image reconstruction from 90-angle projection data, 151 sampled values for each angle. (a) Object function, (b) estimate by the FBP, (c) estimate by the ART after one iteration using the data access order of random permutation, (d) prior function, (e) estimate by the DPDFT after one iteration using the data access order of random permutation and the prior in (d).

\section{References}

1. C. L. Byrne and R. M. Fitzgerald, "Reconstruction from partial information, with applications to tomography," SIAM J. Appl. Math. 42, 933-940 (1982).

2. C. L. Byrne, R. M. Fitzgerald, M. A. Fiddy, T. J. Hall, and A. M. Darling, "Image restoration and resolution enhancement," J. Opt. Soc. Am. 73, 1481-1487 (1983).

3. H. M. Shieh, C. L. Byrne, and M. A. Fiddy, "Image reconstruction: a unifying model for resolution enhancement and data extrapolation. Tutorial," J. Opt. Soc. Am. A 23, 258-266 (2006).

4. H. M. Shieh, C. L. Byrne, M. E. Testorf, and M. A. Fiddy, "Iterative image reconstruction using prior knowledge," J. Opt. Soc. Am. A 23, 1292-1300 (2006).

5. C.L. Byrne and R. M. Fitzgerald, "A unifying model for spectrum estimation," in Proceedings of the RADC Spectrum Estimation Workshop, In-House Report RADC-TR-79-63 (Rome Air Development Center, Griffiss Air Force Base, 1979), pp. 157-162.
6. C. L. Byrne, B. M. Levine, and J. Dainty, "Stable estimation of the probability density function of intensity from photon frequency counts," J. Opt. Soc. Am. A 1, 1132-1135 (1984).

7. C. L. Byrne and R. M. Fitzgerald, "Spectral estimators that extend the maximum entropy and maximum likelihood methods," SIAM J. Appl. Math. 44, 425-442 (1984).

8. C. L. Byrne and M. A. Fiddy, "Estimation of continuous object distributions from limited Fourier magnitude measurements," J. Opt. Soc. Am. A 4, 112-117 (1987).

9. C. L. Byrne and M. A. Fiddy, "Image as power spectra; reconstruction as a Wiener filter approximation,” Inverse Probl. 4, 399-409 (1988).

10. C. L. Byrne, Signal Processing: a Mathematical Approach (A K Peters, 2005).

11. R. Gordon, R. Bender, and G. T. Herman, "Algebraic reconstruction techniques (ART) for three-dimensional electron microscopy and x-ray photography," J. Theor. Biol. 29, 471-481 (1970). 